\title{
Acute Reperfusion Decision-Making in Stroke Patients with Comorbidities: Further Unmasking UNMASK-EVT
}

Keywords: Stroke, Thrombolysis, Endovascular thrombectomy, Medical decision making doi:10.1017/cjn.2020.176

The design of many stroke trials, including those of endovascular therapy (EVT), is set to select relatively uncomplex patients who may be quite different from patients encountered in actual practice. ${ }^{1-6}$ The results of these trials may not always generalize to population groups with a high level of comorbidity or disability. However, such patients make up a significant proportion of those affected by acute stroke in real-life, and clinicians sometimes struggle to understand the risk/benefit analysis which guides the treatment decision. ${ }^{1,2,7-9}$ There are little data available to describe how stroke physicians merge both evidence and patient factors into a treatment choice. ${ }^{9}$

Ganesh et al. explored reperfusion decisions in the setting of such comorbidities in an international cross-sectional web-based survey (UNMASK-EVT). ${ }^{10}$ The study examined 6070 scenariobased responses in a convenience sample of 607 physicians across 38 countries including various physician specialties. They presented respondents with 10 randomly assigned anterior circulation large vessel occlusion (LVO) case scenarios from a pool of 22. Of these, five (1. Stage IV metastatic prostate cancer; 2 . heart failure, chronic obstructive pulmonary disease (COPD), and renal insufficiency requiring dialysis; 3 . non-metastatic prostate cancer with anti-hormonal treatment; 4 . non-disabling/mild cognitive impairment (MCI); and 5. rheumatoid arthritis requiring care in a nursing home but without cognitive impairment) were mutually exclusive comorbid scenarios. Of the remaining 17 scenarios, 6 represented level $1 \mathrm{~A}$ evidence decisions, and the remaining 11 were either backed by $2 \mathrm{~B}$ evidence or the evidence level was "unknown." The study scenarios also included assumptions of decisions under "current resources" or "ideal conditions."

The study was analytically thorough and the results are complex to interpret but worth examining. When different factors are added together in LVO scenarios, there are variable effects on therapy decisions including reversal of initial decisions. The authors outline a number of these points in their discussion. Among 1379 comorbidity-related responses, physicians less often favored endovascular thrombectomy in individual comorbid scenarios, as compared to scenarios with level 1A evidence under current resources ( $80 \%$ vs. $91 \%$ ) and ideal conditions (83\% vs. 95\%). Specifically, they were less likely to choose EVT with both non-metastatic and metastatic cancer, and MCI relative to $1 \mathrm{~A}$ scenarios. When all 22 scenarios were included in a multivariate model after adjustment for other factors, the presence of comorbidities did not consistently affect thrombectomy decisions. Alteplase was generally favored similarly between individual comorbid and standard scenarios $(72 \%-75 \%$ vs.
$69 \%-72 \%$ ), however less likely for metastatic cancer. Respondents reporting more thrombectomy cases/year, and interventional neuroradiologists, were more likely to relinquish alteplase when pursuing thrombectomy.

There were geographic differences in the decision for EVT with current resources, the most striking being South Asia choosing EVT much less than East Asia. Under ideal circumstances, however, this difference was neutralized suggesting clinicians incorporated factors such as availability of funding (including the ability of patient to pay) into their responses. Practicing in East Asia was a significantly higher predictor of choice for EVT relative to North America in either current or ideal conditions. For specific comorbidity scenarios (i.e. physical dependency in a nursing home), respondents practicing in Australia/New Zealand were less likely to choose EVT than those in North America. Similarly, for some scenarios (i.e. metastatic cancer and heart failure/COPD/dialysis), respondents from Europe were less likely to give alteplase than those from North America.

It is concerning that older age and female sex were associated with lower odds of EVT and female sex was associated with lower odds of alteplase. In fact, the combination of older age and female sex may have reduced the likelihood of respondents choosing EVT in the MCI scenario. The direction of decision reversed once age and sex were adjusted suggesting reverse confounding. It's also disappointing to note that less than $16 \%$ of survey respondents were women. This may reflect the composition of stroke physicians internationally and should be a target for future improvement.

It is of interest that the respondents were more likely to choose EVT in comorbidity scenarios where supporting evidence was otherwise level 1A than in non-comorbid scenarios where the evidence level was lower. On average, this suggests that stroke physicians are more likely to be inclusive when applying 1A evidence but more cautious when operating outside of high-level evidence even with otherwise optimal individuals.

The study has some limitations. There are problems with the survey itself when characterizing comorbidity to reflect real practice. Aside from the single cardiac/renal/pulmonary scenario, the study and statistical design did not permit the coexistence of comorbidities in the same patient scenario. However, in real-life, it is probably more likely that acute stroke patients who are otherwise eligible for reperfusion also have various combinations of such comorbidities. ${ }^{9,10}$ While the authors have acknowledged this deficiency in their discussion and included several other 
covariates in their models, this could limit the generalizability of results. ${ }^{11}$ While some of the comorbidities have been addressed in this survey, other relevant ones that may impact clinical decisions are missing. For example, the authors selected a hypothetical scenario with prostate malignancy, which some clinicians may not find as concerning when treating patients as other types of malignancies such as lung cancer. ${ }^{12}$ Lastly, this is a convenience sample selected by international members of the research group. Nonetheless, it had 607 completed surveys and a respectable $45 \%$ completion rate.

In conclusion, the current study, despite its limitations, provides important insights into decision-making in different clinical scenarios that stroke physicians face in real-life. One of the most valuable components of this study is the description of the rate of choice for thrombectomy in various situations including crosscountry comparisons and respondent characteristics. In situations of uncertainty, it can be helpful to consider how an international community of our peers is approaching the same difficult decisions we ourselves are facing.

\section{Disclosures}

The authors declare no conflict of interest.

Ali Z. Nomani

Katz Group, Department of Medicine, Division of Neurology, University of Alberta, Edmonton, Alberta, Canada

Thomas Jeerakathil

Department of Medicine, Division of Neurology, University of Alberta, Edmonton, Alberta, Canada

Correspondence to: Thomas Jeerakathil, Department of Medicine, Division of Neurology, University of Alberta, 7-112G CSB, 11350-83rd Avenue, Edmonton, Alberta, Canada T6G 2G3. Email: thomasj@ualberta.ca

\section{REFERENCES}

1. Ganesh A, Goyal M. Thrombectomy for acute ischemic stroke: recent insights and future directions. Curr Neurol Neurosci Rep. 2018;18(9):59.

2. Uschner D, Hilgers RD, Heussen N. The impact of selection bias in randomized multi-arm parallel group clinical trials. PLoS One. 2018;13(1):e0192065.

3. Goyal M, Menon BK, Zwam WH, et al. Endovascular thrombectomy after large-vessel ischaemic stroke: a meta-analysis of individual patient data from five randomized trials. Lancet. 2016;387:1723-31.

4. Campbell BCV, Majoie CBLM, Albers GW, et al. Penumbral imaging and functional outcome in patients with anterior circulation ischaemic stroke treated with endovascular thrombectomy versus medical therapy: a meta-analysis of individual patientlevel data. Lancet Neurol. 2019;18:46-55.

5. Albers GW, Marks MP, Kemp S, et al. Thrombectomy for stroke at 6 to 16 hours with selection by perfusion imaging. N Engl J Med. 2018;378:708-18.

6. Nogueira RG, Jadhav AP, Haussen DC, et al. Thrombectomy 6 to 24 hours after stroke with a mismatch between deficit and infarct. N Engl J Med. 2018;378:11-21.

7. Quinn TJ, Taylor-Rowan M, Coyte A, et al. Pre-stroke modified Rankin scale: evaluation of validity, prognostic accuracy, and association with treatment. Front Neurol. 2017;8:275.

8. Cappellari M, Bosco M, Forlivesi S, et al. Reasons for exclusion from intravenous thrombolysis in stroke patients admitted to the Stroke Unit. J Thromb Thrombolysis. 2016;42(4):593-9.

9. Saposnik G, Menon BK, Kashani N, et al. Factors associated with the decision-making on endovascular thrombectomy for the management of acute ischemic stroke. Stroke. 2019;50(9): 2441-47.

10. Ganesh A, Kashani N, Ospel JM, et al. Endovascular therapy or alteplase in patients with comorbidities: insights from UNMASK EVT. Can J Neurol Sci. 2020;48(1):77-86.

11. Polissar L, Diehr P. Regression analysis in health services research: the use of dummy variables. Med Care. 1982;20(9):959-66.

12. Valderas JM, Starfield B, Sibbald B, Salisbury C, Roland M. Defining comorbidity: implications for understanding health and health services. Ann Fam Med. 2009;7(4):357-63. 